\title{
Microbiology test result reporting times and association with outcomes of adults hospitalised with unspecified pneumonia: a data linkage study.
}

\author{
Anil Shrestha ${ }^{1}$, Andrew Georgiou ${ }^{2}$, and Nasir Wabe ${ }^{3}$ \\ ${ }^{1}$ Australian Institute of Health Innovation \\ ${ }^{2}$ Centre for Health Systems and Safety Research, Australian Institute of Health Innovation, \\ Macquarie University \\ ${ }^{3}$ Affiliation not available
}

August 7, 2021

\begin{abstract}
Background: Pneumonia is one of the leading causes of mortality and morbidity worldwide. Microbiology tests play a critical role in the diagnosis of pneumonia. Our study aimed to determine microbiology result reporting times and evaluate its association with outcomes of adult patients ([?]18 years) hospitalized with pneumonia. Methods: A 3-year (2016-2018) retrospective cohort study in six hospitals in New South Wales, Australia. Study data were obtained by linking hospital and laboratory system databases. Result reporting times including time from admission to the first and the last microbiology test results were determined. The outcome measures were hospital length of stay (LOS) and in-hospital mortality. We fit median and logistic regression to evaluate the association of time-to-first microbiological result with hospital LOS and in-hospital mortality respectively. Results: A total of 6,298 patients met the inclusion criteria. Of these, $85.3 \%(\mathrm{n}=5,375)$ ordered at least one microbiology test. The top 5 microbiology tests were blood culture, urine culture, respiratory polymerase chain reaction (PCR), urine antigen and sputum culture. The median time-to-first microbiology result was 26 hrs while the median time-to last test result was $144 \mathrm{hrs}$. The rate of in-hospital mortality was $5.9 \%(\mathrm{n}=371)$. After adjusting for confounders, every 5 hrs increase in the time to-first microbiology test was associated with an increase of $3.9 \mathrm{hrs}$ in the median hospital LOS [95\% Confidence Interval (CI), 3.5 to $4.3 ; \mathrm{P}<0.001]$. There was no association between time-to-first microbiology result and in-hospital mortality (OR 1.01; 95\% CI 1.00-1.02; $\mathrm{P}=0.122$ ). Conclusion: Time-to-first microbiology result reporting was significantly associated with hospital LOS but not with in-hospital mortality. Further research should be conducted to understand if improving result reporting times can reduce the length of hospital stay of patients. Key words: Microbiology diagnostics; Test result reporting; Pneumonia; Diagnostic informatics
\end{abstract}

\section{Hosted file}

Main document.docx available at https://authorea.com/users/429474/articles/533161microbiology-test-result-reporting-times-and-association-with-outcomes-of-adultshospitalised-with-unspecified-pneumonia-a-data-linkage-study

\section{Hosted file}

List of Figures.docx available at https://authorea.com/users/429474/articles/533161microbiology-test-result-reporting-times-and-association-with-outcomes-of-adultshospitalised-with-unspecified-pneumonia-a-data-linkage-study

\section{Hosted file}


List of Tables.docx available at https://authorea.com/users/429474/articles/533161microbiology-test-result-reporting-times-and-association-with-outcomes-of-adultshospitalised-with-unspecified-pneumonia-a-data-linkage-study 